\title{
ON JACOB'S CONSTRUCTION OF THE RATIONAL CANONICAL FORM OF A MATRIX*
}

\author{
MEINOLF GECK ${ }^{\dagger}$
}

\begin{abstract}
H.G. Jacob's elegant approach to the rational canonical, or Frobenius normal form of a linear map is presented here in pure matrix language, thereby avoiding the abstract machinery and prerequisites in the original paper. Related algorithmic aspects and an efficient implementation in the computer algebra system GAP are also discussed.
\end{abstract}

Key words. Rational canonical form, Minimal polynomial of a matrix, Computer algebra.

AMS subject classifications. 15A21, 11C99.

1. Introduction. Jacob [6] presents one of the shortest and most elegant approaches to the rational canonical form of a matrix $A$ over an arbitrary field $K$. The argument relies on the abstract dictionary between vector spaces and their duals. It is one purpose of this note to point out that, when dressed in pure matrix language, the argument becomes particularly simple, short and transparent, where no consideration of any duality operation is required; see Section 3. Once this is done, a strengthening of the whole argument presents itself quite naturally. Namely, a further prerequisite in [6] is the existence of vectors whose minimal polynomial equals the global minimal polynomial of $A$. (The usual proofs of this fact require some arguing and rely on the prime factorisation of polynomials; see, for example, [6, §2 (i)], [1, 8.13] or [9].) We shall see that we do not need this existence result as a prerequisite any more, but can simply deduce it as a very easy corollary.

As pointed out in Bongartz [4] or Ballester-Bolinches et al. [3], one can also produce such vectors by an algorithm which only relies on the usual matrix operations and the Euclidean algorithm for polynomials. In Section 4, we revise these arguments and discuss some further algorithmic issues.

A new GAP [5] program, based on this note and incorporating all these ingredients, including Jacob's construction [6] and the Neunhöffer-Praeger algorithm [7] for computing minimal polynomials of matrices, is available at the author's webpage at https://pnp.mathematik.uni-stuttgart.de/iaz/iaz2/geckmf/; it appears to perform well even on the large test matrices in [7].

We have tried to keep this note as elementary as possible and, hence, to make it suitable for inclusion in a basic Linear Algebra course. (In fact, it was motivated by teaching such a course.)

2. Preliminaries. Let us fix some notation. Let $M_{n}(K)$ be the vector space of $n \times n$ matrices over $K$. Let $K^{n}$ be the vector space of column vectors of length $n$. Let $\left\{e_{1}, \ldots, e_{n}\right\}$ be the standard basis of $K^{n}$. Let $K[X]$ be the polynomial ring over $K$ with indeterminate $X$.

The minimal polynomial of $A \in M_{n}(K)$ is the unique monic polynomial $f \in K[X]$ of smallest possible

${ }^{*}$ Received by the editors on January 24, 2019. Accepted for publication on January 29, 2020. Handling Editor: Bryan L. Shader.

${ }^{\dagger}$ IAZ - Lehrstuhl für Algebra, Universität Stuttgart, Pfaffenwaldring 57, 70569 Stuttgart, Germany (meinolf.geck@mathematik.uni-stuttgart.de). 
degree such that $f(A)=0$; it will be denoted by $\mu_{A}$. The minimal polynomial of a vector $v \in K^{n}$ is the unique monic polynomial $f \in K[X]$ of smallest possible degree such that $f(A) v=0$; it will be denoted by $\mu_{A, v}$. It is well-known and easy to see that $\mu_{A, v} \mid \mu_{A}$ for all $v \in K^{n}$. Note that, if $v \in K^{n}$ and $d:=\operatorname{deg}\left(\mu_{A, v}\right) \geq 1$, then the subspace

$$
U_{A, v}:=\left\langle v, A v, A^{2} v, \ldots, A^{d-1} v\right\rangle_{K} \subseteq K^{n}
$$

is $A$-invariant and has dimension $d$. Furthermore, since $\mu_{A, v}(A) v=0$, it immediately follows that $\mu_{A, v}(A) x=$ 0 for all $x \in U_{A, v}$. For all this see, for example, [1, § 8.2].

Of particular importance is the case where $\operatorname{dim} U_{A, v}$ is as large as possible. We say that $v_{0} \in K^{n}$ is a maximal vector if

$$
\operatorname{deg}\left(\mu_{A, v_{0}}\right)=\max \left\{\operatorname{deg}\left(\mu_{A, v}\right) \mid v \in K^{n}\right\} .
$$

(We shall see in Section 3 that $\mu_{A, v_{0}}=\mu_{A}$ for any maximal vector $v_{0}$.)

Finally, let $f=a_{0}+a_{1} X+\cdots+a_{n-1} X^{n-1}+X^{n} \in K[X]$ be a monic polynomial of degree $n$. The corresponding companion matrix is given by

$$
A_{f}:=\left[\begin{array}{ccccc}
0 & 0 & \cdots & 0 & -a_{0} \\
1 & 0 & \cdots & 0 & -a_{1} \\
0 & 1 & \cdots & 0 & -a_{2} \\
\vdots & \vdots & \ddots & \vdots & \vdots \\
0 & 0 & \cdots & 1 & -a_{n-1}
\end{array}\right] \in M_{n}(K)
$$

Thus, $A_{f} e_{n}=-\left(a_{0} e_{1}+a_{1} e_{2}+\cdots+a_{n-1} e_{n}\right)$ and $A_{f} e_{i}=e_{i+1}$ for $1 \leq i \leq n-1$. Note that the first standard basis vector $e_{1}$ is a maximal vector for $A_{f}$.

3. The basic construction according to Jacob. Let $A \in M_{n}(K)$. Take any non-zero $v_{0} \in K^{n}$ and let $f:=\mu_{A, v_{0}} \in K[X]$ be the minimal polynomial of $v_{0}$; we set $d:=\operatorname{deg}(f) \geq 1$. We can extend the $d$ linearly independent vectors $v_{0}, A v_{0}, \ldots, A^{d-1} v_{0}$ to a basis of $K^{n}$. With respect to this new basis, the linear operator on $K^{n}$ defined by $A$ is represented by a matrix in block triangular shape as follows

$$
T:=\left(\begin{array}{c|c}
A_{f} & * \\
\hline 0 & B
\end{array}\right) \in M_{n}(K), \quad \text { where } B \in M_{n-d}(K) .
$$

Since $A$ and $T$ represent the same linear operator on $K^{n}$ with respect to different bases, we certainly have $\mu_{A}=\mu_{T}$. Also note that, now, $f=\mu_{T, e_{1}}$ is the minimal polynomial of $e_{1}$ with respect to $T$; furthermore, $v_{0}$ is a maximal vector for $A$ if and only if $e_{1}$ is a maximal vector for $T$. The next and crucial step is to try to find a $T$-invariant complementary subspace to the $T$-invariant subspace $\left\langle e_{1}, \ldots, e_{d}\right\rangle_{K}$ of $K^{n}$ (on which $T$ acts via $\left.A_{f}\right)$. For any $x \in K^{n}$, we denote by $[x]_{d} \in K$ the $d$-th component of $x$.

Proposition 3.1. (Cf. Jacob $[6, \S 3]$ ) With the above notation, let

$$
W:=\left\{x \in K^{n} \mid\left[T^{j-1} x\right]_{d}=0 \quad \text { for } 1 \leq j \leq d\right\} .
$$

Then $W$ is a subspace of $K^{n}$ such that $K^{n}=\left\langle e_{1}, \ldots, e_{d}\right\rangle_{K} \oplus W$. If $e_{1}$ is a maximal vector for $T$, then $W$ is T-invariant. 
Proof. Each of the $d$ equations $\left[T^{j-1} x\right]_{d}=0$ is a homogeneous linear equation for the components of $x$. Thus, $W$ is defined by a system of $d$ linear equations, and so $\operatorname{dim} W \geq n-d$. Next we show that $W \cap\left\langle e_{1}, \ldots, e_{d}\right\rangle_{K}=\{0\}$. Let $x \in W$ and assume that $x=s_{1} e_{1}+\cdots+s_{d} e_{d}$, where $s_{i} \in K$. First, since $x \in W$, we certainly have $s_{d}=[x]_{d}=\left[T^{0} x\right]_{d}=0$. Next, since $T e_{i}=e_{i+1}$ for $1 \leq i \leq d-1$, we obtain that $T x=s_{1} e_{2}+\cdots+s_{d-1} e_{d}$. If $d>1$, we conclude again that $s_{d-1}=[T x]_{d}=0$. Continuing in this way with $T^{2} x, T^{3} x, \ldots, T^{d-1} x$, we find that $s_{i}=0$ for all $i$, and so $x=0$. Hence, $K^{n}=\left\langle e_{1}, \ldots, e_{d}\right\rangle_{K} \oplus W$.

Now assume that $e_{1}$ is a maximal vector for $T$. Let $x \in W$; we must show that $T x \in W$. Now, let $1 \leq j \leq d$ and $d^{\prime}:=\operatorname{deg}\left(\mu_{T, x}\right) \geq 1$; by assumption, we have $d^{\prime} \leq d$. Then $\left[T^{j-1}(T x)\right]_{d}=\left[T^{j} x\right]_{d}$ and $T^{j} x \in\left\langle x, T x, \ldots, T^{d^{\prime}-1} x\right\rangle_{K}$; writing $T^{j} x$ as a linear combination of $x, T x, \ldots, T^{d^{\prime}-1} x$, we see that $\left[T^{j} x\right]_{d}=0$. Thus, $W$ is $T$-invariant.

Corollary 3.2. Assume that $e_{1}$ is a maximal vector for $T$. Then $\mu_{T, e_{1}}=\mu_{T}$.

(Consequently, we also have $\mu_{A, v_{0}}=\mu_{A}$ if $v_{0}$ is a maximal vector for $A$.)

Proof. Let $f=\mu_{T, e_{1}} \in K[X]$ as above, and $W \subseteq K^{n}$ as in Proposition 3.1. Since $\left\langle e_{1}, \ldots, e_{d}\right\rangle_{K}=U_{T, e_{1}}$ (see Section 2), we have $f(T) x=0$ for all $x \in\left\langle e_{1}, \ldots, e_{d}\right\rangle_{K}$. So it is enough to show that $f(T) w=0$ for all $w \in W$. Now, given $w \in W$, we set $h:=\mu_{T, e_{1}+w} \in K[X]$. Then $h(T)\left(e_{1}+w\right)=0$ and so $h(T) e_{1}=-h(T) w$. The left-hand side lies in $\left\langle e_{1}, \ldots, e_{d}\right\rangle_{K}$ and the right-hand side lies in $W$. Hence, we must have $h(T) e_{1}=0$ and $h(T) w=0$. It follows that $f \mid h$. Since $e_{1}$ is a maximal vector for $T$, we have $\operatorname{deg}(h) \leq \operatorname{deg}(f)$. This forces $f=h$ and, hence, $f(T) w=0$.

Assume, as above, that $e_{1}$ is a maximal vector for $T$ and let $w_{1}, \ldots, w_{n-d}$ be a basis of $W$. Then $e_{1}, \ldots, e_{d}, w_{1}, \ldots, w_{n-d}$ is a new basis of $K^{n}$ and, with respect to this new basis, the linear operator on $K^{n}$ defined by $T$ is represented by a matrix in block diagonal form as follows

$$
\left(\begin{array}{c|c}
A_{f} & 0 \\
\hline 0 & A^{\prime}
\end{array}\right), \quad \text { where } A^{\prime} \in M_{n-d}(K) .
$$

By Corollary 3.2, we have $f\left(A^{\prime}\right)=0$ and so $\mu_{A^{\prime}} \mid f$. Applying an inductive argument to $A^{\prime}$, we eventually obtain a matrix in block diagonal form

$$
\tilde{A}=\left(\begin{array}{cccc}
A_{f_{1}} & 0 & \cdots & 0 \\
0 & A_{f_{2}} & \ddots & \vdots \\
\vdots & \ddots & \ddots & 0 \\
0 & \cdots & 0 & A_{f_{r}}
\end{array}\right)
$$

where $f=f_{1}, f_{2}, \ldots, f_{r} \in K[X]$ are monic polynomials such that $f_{i+1} \mid f_{i}$ for $1 \leq i \leq r-1$. Thus, the above matrix $\tilde{A}$ really is the rational canonical form of $A$. (The unicity of the rational canonical form is easily proved as in [4, Lemma 3$]$, by a rank consideration.)

REMARK 3.3. The above procedure yields an algorithm for constructing $\tilde{A}$, which involves the following computational tasks.

(1) Compute a maximal vector $v \in K^{n}$ for $A$ and the polynomial $\mu_{A, v}$.

(2) Perform the base change transformation from $A$ to $T$ as above.

(3) Compute complementary subspaces $W$ as in Proposition 3.1. 
In Section 4, we comment on (1). The task in (2) is standard Linear Algebra. To solve (3), note that $\left[T^{j-1} x\right]_{d}=e_{d}^{\operatorname{tr}}\left(T^{j-1} x\right)=\left(e_{d}^{\operatorname{tr}} T^{j-1}\right) x$ for any $x \in K^{n}$ and $1 \leq j \leq d$. Hence, if $F$ denotes the $d \times n$ matrix with rows given by $e_{d}^{\operatorname{tr}} T^{j-1}$ for $1 \leq j \leq d$, then $W=\left\{x \in K^{n} \mid F x=0\right\}$. Thus, (3) reduces to the computation of the null space of a matrix.

Remark 3.4. Assume that $A$ is nilpotent. Then $\mu_{A, v} \in K[X]$ is a power of $X$ for any $v \in K^{n}$. Now note that $A_{X^{m}}$, for any $m \geq 1$, is just a Jordan block with eigenvalue 0 . Hence, the above procedure yields a particularly efficient proof for the Jordan normal form of $A$.

4. Maximal vectors and the minimal polynomial. Recall that a maximal vector for $A \in M_{n}(K)$ is a vector $v_{0} \in K^{n}$ such that the degree of $\mu_{A, v_{0}} \in K[X]$ is as large as possible. We now briefly discuss the algorithmic problem of finding such a vector $v_{0}$. As indicated in Section 1, we will do this by avoiding any appeal to the prime factorisation of polynomials.

Let $a, b \in K[X]$ be non-zero. Then the Euclidean Algorithm yields the existence of a unique monic greatest common divisior $d=\operatorname{gcd}(a, b) \in K[X]$, as well as polynomials $r, s \in K[X]$ such that $d=r a+s b$. This is all we require in the following discussion. Here is a simple illustration.

REMARK 4.1. We say that non-zero $a, b \in K[X]$ are coprime if $\operatorname{gcd}(a, b)=1$. Assume that this is the case, and let $r, s \in K[X]$ be such that $1=r a+s b$.

(a) If also $c \in K[X]$ is non-zero and $\operatorname{gcd}(a, c)=1$, then $\operatorname{gcd}(a, b c)=1$.

(b) For any $m \in \mathbb{Z}_{\geq 1}$, we also have $\operatorname{gcd}\left(a, b^{m}\right)=1$.

(c) If $c \in K[X]$ and $a \mid b c$, then $a \mid c$.

To prove (a), let $u, v \in K[X]$ such that $1=u a+v c$. Then $1=(r a+s b)(u a+v c)=(r u a+r v c+s b u) a+(s v) b c$. To get (b), apply (a) repeatedly with $c=b$. For (c), note that $a \mid r a c+s b c=c$.

Lemma 4.2. Let $a, b \in K[X]$ be non-zero. Set $c:=\operatorname{gcd}\left(b^{\operatorname{deg}(a)}, a\right) \in K[X]$ and write $a=c a^{\prime}$ with $a^{\prime} \in K[X]$. Then $\operatorname{gcd}\left(a^{\prime}, b\right)=1$.

Proof. Let $d:=\operatorname{gcd}\left(a^{\prime}, b\right)$ and assume, if possible, that $\operatorname{deg}(d) \geq 1$. Since $a^{\prime} \mid a$, we have $d \mid a$. Let $i \geq 1$ be the largest integer such that $d^{i} \mid a$. Then $i \leq \operatorname{deg}\left(d^{i}\right) \leq \operatorname{deg}(a)$. On the other hand, since $d \mid b$, we conclude that $d^{i} \mid b^{i}$ and, hence, $d^{i} \mid b^{\operatorname{deg}(a)}$. Thus, we have $d^{i} \mid \operatorname{gcd}\left(b^{\operatorname{deg}(a)}, a\right)=c$. But then $d^{i+1}=d^{i} d \mid c a^{\prime}=a$, contradiction to the definition of $i$.

The following result, taken from [4], shows that a least common multiple of two non-zero polynomials $a, b \in K[X]$ can always be written as the product of two coprime factors, one of which divides $a$ and the other one divides $b$. In [4], a proof is sketched based on the prime factorisation of polynomials in $K[X]$. Our argument avoids that completely. (A similar result appears in $[3, \S 2]$ and, implicitly, also in [9, Lemma 2].)

Lemma 4.3. (Bongartz [4, Lemma 5]) Let $a, b \in K[X]$ be non-zero. Let $d=\operatorname{gcd}(a, b)$ and write $a=d \tilde{a}$, $b=d \tilde{b}$ with $\tilde{a}, \tilde{b} \in K[X]$. (Thus, dãa is a least common multiple of $a, b$.) Set $b_{1}:=\operatorname{gcd}\left(\tilde{b}^{\operatorname{deg}(b)}, b\right)$ and $a_{1}:=\tilde{a} b^{\prime}$, where $b^{\prime} \in K[X]$ is such that $b=b^{\prime} b_{1}$. Then

$$
a_{1} b_{1}=d \tilde{a} \tilde{b}, \quad a_{1}\left|a, \quad b_{1}\right| b \quad \text { and } \quad \operatorname{gcd}\left(a_{1}, b_{1}\right)=1 .
$$

Proof. We verify that $a_{1}, b_{1}$ have the desired properties. First, $b_{1} \mid b$ and $a_{1} b_{1}=\tilde{a} b^{\prime} b_{1}=d \tilde{a} \tilde{b}$. Next, $\operatorname{gcd}(\tilde{a}, \tilde{b})=1$ by the definition of $\tilde{a}, \tilde{b}$. By Lemma 4.2 , we also have $\operatorname{gcd}\left(b^{\prime}, \tilde{b}\right)=1$. So Remark 4.1(a) yields that $\operatorname{gcd}\left(a_{1}, \tilde{b}\right)=\operatorname{gcd}\left(\tilde{a} b^{\prime}, \tilde{b}\right)=1$. Since $a_{1} \mid d \tilde{a} \tilde{b}$, this implies that $a_{1} \mid d \tilde{a}=a$; see Remark 4.1(c). Now 
consider $\operatorname{gcd}\left(a_{1}, b_{1}\right)$. Since $\operatorname{gcd}\left(a_{1}, \tilde{b}\right)=1$, we also have $\operatorname{gcd}\left(a_{1}, \tilde{b}^{\operatorname{deg}(b)}\right)=1$; see Remark $4.1(\mathrm{~b})$. On the other hand, $b_{1}=\operatorname{gcd}\left(\tilde{b}^{\operatorname{deg}(b)}, b\right) \mid \tilde{b}^{\operatorname{deg}(b)}$ and so we conclude that $\operatorname{gcd}\left(a_{1}, b_{1}\right)=1$.

Finally, let us recall from [3], [4] and [9] how the above result leads to a solution for the problem of finding maximal vectors. The following two properties easily follow from the definition of the minimal polynomial of a vector; see, e.g., [4, Lemma 6].

(1) If $v \in K^{n}$ and $\mu_{A, v}=f g$, where $f, g \in K[X]$, then $\mu_{A, f(A) v}=g$.

(2) If $v, w \in K^{n}$ and $\operatorname{gcd}\left(\mu_{A, v}, \mu_{A, w}\right)=1$, then $\mu_{A, v+w}=\mu_{A, v} \mu_{A, w}$.

We obtain the following immediate consequence. Note that the proof provides an explicit construction of the new vector $z$.

LEMmA 4.4. Let $v, w \in K^{n}$. Then there exists some $z \in K^{n}$ such that $\mu_{A, v} \mid \mu_{A, z}$ and $\mu_{A, w} \mid \mu_{A, z}$.

Proof. Let $d=\operatorname{gcd}\left(\mu_{A, v}, \mu_{A, w}\right)$ and write $\mu_{A, v}=d f, \mu_{A, w}=d g$, where $f, g \in K[X]$. Applying Lemma 4.3 to $\mu_{A, v}, \mu_{A, w}$, we obtain $f_{1}, g_{1} \in K[X]$ such that $f_{1}\left|\mu_{A, v}, g_{1}\right| \mu_{A, w}, \operatorname{gcd}\left(f_{1}, g_{1}\right)=1$ and $f_{1} g_{1}=d f g$. Now set

$$
z:=\tilde{f}(A) v+\tilde{g}(A) w \in K^{n}
$$

where $\tilde{f}, \tilde{g} \in K[X]$ are such that $\mu_{A, v}=f_{1} \tilde{f}$ and $\mu_{A, w}=g_{1} \tilde{g}$. Using the above two properties (1) and (2), we find that $\mu_{A, z}=f_{1} g_{1}$.

As a by-product, the following result yields yet another proof for the fact that $\mu_{A}$ is the minimal polynomial of a maximal vector for $A$.

COROllary 4.5. (Bongartz [4, Lemma 6]) There is an effective algorithm (which only requires the usual matrix operations, including Gaussian elimination, and gcd computations in $K[X])$ for finding a maximal vector for $A$. If $v_{0} \in K^{n}$ is any maximal vector for $A$, then $\mu_{A, v_{0}}=\mu_{A}$.

Proof. We describe a procedure for obtaining a maximal vector. Set $z_{1}:=0$ and consider the standard basis $\left\{e_{1}, \ldots, e_{n}\right\}$ of $K^{n}$. Applying Lemma 4.4 repeatedly, we obtain a sequence of vectors $z_{2}, \ldots, z_{n} \in K^{n}$ such that $\mu_{A, z_{i-1}} \mid \mu_{A, z_{i}}$ and $\mu_{A, e_{i}} \mid \mu_{A, z_{i}}$ for $2 \leq i \leq n$. Consequently, $\mu_{A, e_{i}} \mid \mu_{A, z_{n}}$ for all $i$ and, hence, $\mu_{A, z_{n}}(A) v=0$ for all $v \in K^{n}$, which means that $\mu_{A, z_{n}}(A)=0$. Since $\mu_{A, v} \mid \mu_{A}$ for any $v \in K^{n}$, we conclude that $\mu_{A}=\mu_{A, z_{n}}$ and that $z_{n}$ is a maximal vector for $A$.

Finally, let $v_{0} \in K^{n}$ be an arbitrary maximal vector. Then we have $\operatorname{deg}\left(\mu_{A, v_{0}}\right)=\operatorname{deg}\left(\mu_{A, z_{n}}\right)=\operatorname{deg}\left(\mu_{A}\right)$. Since also $\mu_{A, v_{0}} \mid \mu_{A}$, this forces that $\mu_{A, v_{0}}=\mu_{A}$.

There are some straightforward optimisations of the procedure in the above proof (for example, one does not have to use all standard basis vectors $e_{i}$ but rather work along a chain of cyclic subspaces). In any case, based on Corollary 4.5 and the construction of $W$ in Proposition 3.1, one obtains an algorithm for computing maximal vectors, their minimal polynomials and, finally, the rational canonical form of a matrix which works for matrices over any field $K$. An implementation of that algorithm in GAP [5] can be found on the author's website; the main functions it provides are MaximalVectorMat and FrobeniusNormalForm. As far as we are aware of, this is the first efficient implementation incorporating all of the above features.

Now assume, finally, that $K$ is a finite field with $q$ elements. Then one motivation for making the above procedures as efficient as possible comes from applications in finite group theory; see, for example, Bäärnhielm et al. $[2, \S 1]$. The currently best algorithm for computing $\mu_{A}$ is probably that in NeunhöfferPraeger [7, Theorem 1.1]. Its efficiency analysis relies on the difficulty of computing the prime factorisation 
of polynomials, which becomes an issue if $q$ is large (see [7, Remark 3.3]). The above approach based on the Euclidean algorithm alone would avoid this issue; a similar remark applies to the algorithm in Steel [8].

But note that the Neunhöffer-Praeger algorithm does not output a maximal vector. Therefore, we have also written a function NPMaximalVectorMat which first obtains $\mu_{A}$ from the existing GAP implementation of [7] (available via the package $\mathrm{cvec}$ ), and then determines a maximal vector. For this purpose, we follow again the basic strategy in the proof of Corollary 4.5, but note that we can stop the iteration over the basis vectors $e_{i}$ as soon as a vector $z_{i}$ is found such that the degree of $\mu_{A, z_{i}}$ equals the already known degree of $\mu_{A}$. Applied to the - quite challenging - test matrix

$$
A=M_{2} \in M_{4370}(K) \quad\left(\text { with } K=\mathbb{F}_{2}, \text { see }[7, \S 9.2(\mathrm{~b})]\right),
$$

this yields $\mu_{A}$ and a maximal vector in about 10 seconds. Here, $\mu_{A}$ has degree 2097, with irreducible factors of degrees $1,2,4,6,88,197,854,934$. Once there is an efficient procedure to obtain maximal vectors, the computation of the Frobenius normal form proceeds with a simple recursion (using Jacob's complement in Proposition 3.1). For example, the normal form for $A=M_{2}$ is obtained in less than 90 seconds; it has 2212 diagonal blocks, one of size 2097, one of size 5, one of size 3, a further 56 blocks of size 2 and, finally, 2153 blocks of size 1. Our program also returns an invertible matrix performing the base change and, thus, the correctness of the canonical form computation can be verified independently.

\section{REFERENCES}

[1] T. Andreescu. Essential Linear Algebra with Applications. A Problem-Solving Approach. Springer, New York, 2014.

[2] H. Bäärnhielm, D. Holt, C.R. Leedham-Green, and E.A. O'Brien. A practical model for computation with matrix groups. J. Symbolic Comput., 68:27-60, 2015. Available at http://dx.doi.org/10.1016/j.jsc.2014.08.006.

[3] A. Ballester-Bolinches, R. Esteban-Romero, and V. Pérez-Calabuig. A note on the rational canonical form of an endomorphism of a vector space of finite dimension. Oper. Matrices, 12:823-836, 2018. Available at https: //doi.org/10.7153/oam-2018-12-49.

[4] K. Bongartz. A direct approach to the rational normal form. Preprint, arXiv:1410.1683, 2014. Available at https: //arxiv.org/abs/1410.1683.

[5] The GAP Group. GAP - Groups, Algorithms, and Programming. Version 4.10.0, 2018. Available at http://www. gap-system.org.

[6] H.G. Jacob. Another proof of the rational decomposition theorem. Amer. Math. Monthly, 80:1131-1134, 1973. Available at https://doi.org/10.1080/00029890.1973.11993470.

[7] M. Neunhöffer and C.E. Praeger. Computing minimal polynomials of matrices. LMS J. Comput. Math., 11:252-279, 2008. Available at https://doi.org/10.1112/S1461157000000590.

[8] A. Steel. A new algorithm for the computation of canonical forms of matrices over fields. J. Symbolic Comput., 24: 409-432, 1997. Available at https://doi.org/10.1006/jsco.1996.0142.

[9] D. Zheng and H. Liu. On the minimal polynomial of a vector. Advances in Linear Algebra 83 Matrix Theory, 2:48-50, 2012. Available at http://dx.doi.org/10.4236/alamt.2012.24008. 